vessels were significantly smaller (mean diameter: FOP $18 \pm 2$, NSIP $13 \pm 1$, UIP $13 \pm 1 \mu \mathrm{m})$ compared to controls $(23 \pm 2 \mu \mathrm{m})$. Density of lymphatic vessels was significantly reduced in NSIP and UIP $(21 \pm 2$ $\mathrm{mm}^{-2}$ ), compared to controls $\left(35 \pm 4 \mathrm{~mm}^{-2}\right)$ and their size was significantly greater (mean diameter: NSIP $111 \pm 10 \mu \mathrm{m}$, UIP $121 \pm 5 \mu \mathrm{m}$, controls: $74 \pm 9 \mu \mathrm{m})$. In controls, $85 \pm 6 \%$ of the parenchymal lymphatics were close $(<100 \mu \mathrm{m})$ to a blood vessel, and only $5 \pm 4 \%$ were in proximity of bronchoalveolar spaces, while in all three disease groups they were less frequently perivascular (FOP $47 \pm 6 \%$, NSIP $55 \pm 3 \%$, UIP $56 \pm 2 \%$ ) and more frequently associated with the bronchoalveolar lumen (FOP $52 \pm 11 \%$, NSIP $85 \pm 3 \%$, UIP $69 \pm 2 \%$ ). Lymphatic vessels were rarely seen inside Masson bodies and never inside fibroblastic foci (Abstract S114 figure 1). These data are consistent with a substantial remodelling of lymphatic vessels in fibrotic lung disease, with a shift of lymphatics away from blood vessels.

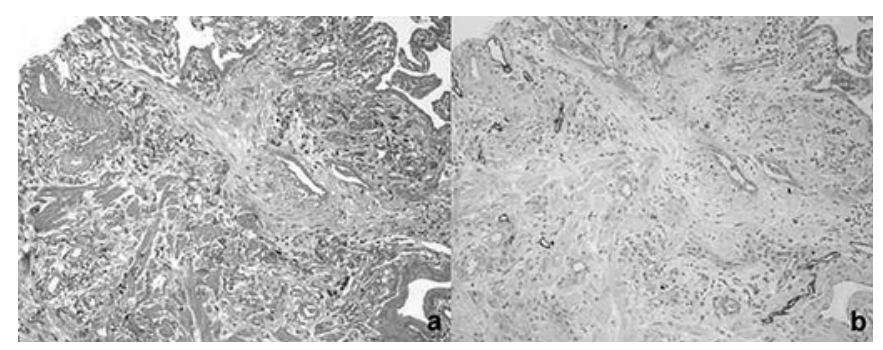

Abstract S114 Figure 1 Two consecutive sections of a VIP biopsy. Movat's pentachrome staining $(A)$ shows fibroblastic focus stained in light blue. Panel B corresponds to the consecutive section, lymphatic vessels stained by D2-40 (black), blood vessels (vWf) in red. Lymphatic vessels are absent within fibroblastic focus, but are seen in the fibrotic interstitium.

\section{Respiratory muscles, exercise and ventilation S115 THE EFFECT OF POSTURE ON THE 2ND INTERCOSTAL SPACE SURFACE PARASTERNAL ELECTROMYOGRAM (EMGPARA): VALIDATING A NOVEL CLINICAL TOOL TO MEASURE NEURAL RESPIRATORY DRIVE}

\section{doi:10.1136/thoraxinl-2011-201054b.115}

${ }^{1} \mathrm{M}$ C Ramsay, ${ }^{1} \mathrm{E}-\mathrm{S}$ Suh, ${ }^{2} \mathrm{~S}$ Mandal, ${ }^{1} \mathrm{P}$ Murphy, ${ }^{2} \mathrm{~J}$ Steier, ${ }^{3} \mathrm{~A}$ Simonds, ${ }^{4} \mathrm{~N}$ Hart. ${ }^{1}$ Department of Asthma, Allergy and Respiratory Science, Division of Asthma, Allergy and Lung Biology, King's College London, London, UK; ${ }^{2}$ Lane Fox Respiratory Unit, Guy's and St Thomas' NHS Foundation Trust, London, UK; ${ }^{3}$ National Heart and Lung Institute, Respiratory Biomedical Research Unit, Royal Brompton Hospital and Imperial College London, London, UK; ${ }^{4}$ Guy's and St Thomas' NHS Foundation Trust and King's College London, National Institute of Health Research Comprehensive Biomedical Research Centre, London, UK

Introduction Although neural respiratory drive (NRD), as measured by diaphragm EMG, has been shown to reflect the balance between the respiratory muscle load and capacity providing a marker of disease severity, it requires insertion of an oesophageal catheter which limits its clinical utility. 2nd intercostal space surface EMGpara has been shown to be a useful alternative non-invasive monitoring tool in acute COPD (Murphy et al. Thorax 2011;66:602-8) and overnight in asthmatic patients (Steier et al. Thorax 2011;66:609-14). Previous data has suggested that there is reduced activation of the chest wall muscles in normal subjects in the supine posture as a consequence of a change in chest wall configuration. To assess the clinical utility and validity of EMGpara to continuously monitor changes in $\mathrm{NRD}$, we investigated the effect of different posture on the EMGpara in normal subjects.

Methods Wet gel electrodes were placed at the parasternal edge of the 2 nd intercostal space following skin preparation. Signals were amplified and filtered before analogue to digital conversion and digital processing providing the raw signal and root mean squared data. Five positions included sitting at 45 degrees, lying flat, lying on the right and left hand side and sitting at 90 degrees. EMGpara was measured during 2 min of tidal breathing in each posture. Resting EMG signal was normalised to the maximal inspiratory manoeuvres performed in each position (EMGpara\%max).

Results Eight healthy subjects were recruited with a mean age 32 years \pm 2 years; 4 male; BMI $23 \pm 2 \mathrm{~kg} / \mathrm{m}^{2}$. Mean EMGpara $\%$ max was $4.60 \pm 3.93 \%$ sitting at 45 degrees, $4.82 \pm 2.27 \%$ lying flat, $5.32 \pm 3.91 \%$ lying on the right hand side, $4.47 \pm 4.47 \%$ lying on the left hand side, $4.58 \pm 3.75 \%$ sitting at 90 degrees. A repeated measures ANOVA showed there was no significant difference in EMG ${ }^{\text {paramax }}$ between the different postures ( $p=0.97$; Abstract S115 figure 1).

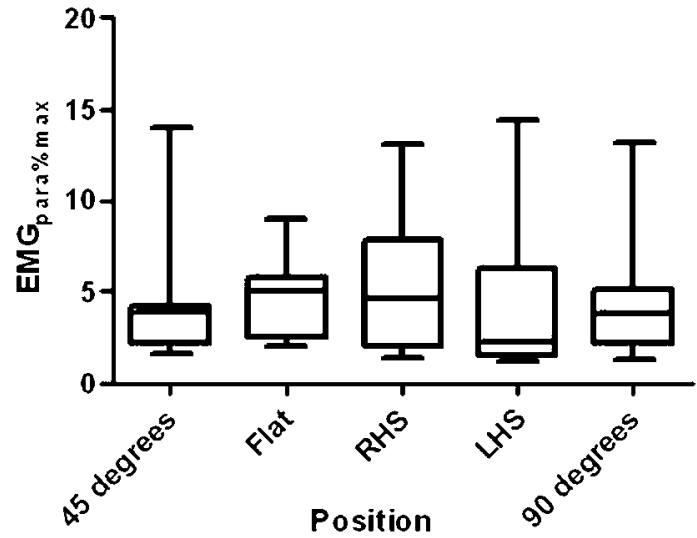

Abstract S115 Figure 1 Box and whisker Plot. Reference position is 45 degrees. There was no difference lying flat $(p=0.9)$, lying on the right hand side (RHS; $p=0.7$ ), lying on the left hand side (LHS; $p=0.9$ ) and with 90 degrees upright $(\mathrm{p}=1.0)$.

Conclusion NRD, as measured by $\mathrm{EMG}_{\text {paramax }}$, is stable across a range of different postures. It provides comparable information independent of body position and could be considered as a monitoring tool in clinical practice, including for overnight monitoring.

\section{S116 PARASTERNAL MUSCLE ELECTROMYELOGRAPHY (EMGpara) REFLECTS OBSERVED CHANGES IN DYNAMIC HYPERINFLATION DURING ACUTE EXACERBATIONS OF CHRONIC OBSTRUCTIVE PULMONARY DISEASE (AECOPD)}

doi:10.1136/thoraxjnl-2011-201054b.116

${ }^{1} \mathrm{E}$ Suh, ${ }^{1} \mathrm{M}$ Ramsay, ${ }^{2} \mathrm{~S}$ Mandal, ${ }^{2} \mathrm{E}$ Boleat, ${ }^{3} \mathrm{~B}$ Christian, ${ }^{3} \mathrm{~K}$ Henderson, ${ }^{1} \mathrm{P}$ Murphy, ${ }^{1} \mathrm{~J}$ Moxham, ${ }^{1,2,4} \mathrm{~N}$ Hart. ${ }^{1}$ Department of Asthma, Allergy and Respiratory Science, Division of Asthma, Allergy and Lung Biology, King's College London, London, UK; ${ }^{2}$ Lane Fox Respiratory Unit, Guy's and St Thomas' NHS Foundation Trust, London, UK; ${ }^{3}$ Emergency Department, Guy's and St Thomas' NHS Foundation Trust, London, UK; ${ }^{4}$ Guy's and St Thomas' NHS Foundation Trust and King's College London, National Institute of Health Research Comprehensive Biomedical Research Centre, London, UK

Background During AECOPD, expiratory flow limitation results in dynamic hyperinflation $(\mathrm{DH})$, respiratory neuromechanical uncoupling, and increased work of breathing causing breathlessness. We have previously demonstrated that 2 nd intercostal space $E M G_{\text {para }}$, as a direct marker of neural respiratory drive (NRD), is able to detect clinical change in hospitalised AECOPD patients. We hypothesised that $\mathrm{EMG}_{\text {para }}$ has an indirect relationship with $\mathrm{DH}$.

Method Patients admitted with AECOPD at a metropolitan teaching hospital were recruited. Inspiratory capacity (IC) was used as a measure of $\mathrm{DH}$. EMG $\mathrm{Eara}_{\text {a }}$ spirometry and IC manoeuvres were measured twice daily from admission until the patient was fit for discharge. Dyspnoea scores (modified Borg score, visual analogue 
scale and numerical rating scale) were recorded. NRD was expressed as $E M G_{\text {paramax }}$ : inspiratory $E M G_{\text {para }}$ signal normalised to a maximum signal measured during a sniff manoeuvre. The changes in $\mathrm{EMG}_{\text {para\%max }}\left(\right.$ ? $\left.\mathrm{EMG}_{\text {para\%max }}\right)$ and IC $(\Delta \mathrm{IC})$ between admission and discharge were analysed.

Results 31 patients were recruited; only 20 (65\%) were able to perform spirometry and IC manoeuvres. The baseline characteristics were $69 \pm 11$ years; male $55 \%$; body mass index $26.1 \pm 7.3 \mathrm{~kg} / \mathrm{m}^{2}$; \% predicted $\mathrm{FEV}_{1} 36.3 \pm 9.3$; and $41 \pm 24$ smoking pack years. The overall mean $\Delta \mathrm{EMG}_{\text {para\%max }}$ of the 20 patients fell by $4 \%$, with $16(80 \%)$ patients experiencing a fall in $\mathrm{EMG}_{\text {paramax }}$ during their admission. We observed an indirect relationship between $\Delta \mathrm{EMG}_{\text {para } \% \max }$ and $\Delta \mathrm{IC}(\mathrm{r}=-0.52, \mathrm{p}=0.02)$, and between $\Delta \mathrm{EMG}_{\mathrm{para} \% \max }$ and $\Delta \mathrm{FVC}$ $(\mathrm{r}=-0.585, \mathrm{p}=0.036)$. We found a correlation between $\Delta \mathrm{IC}$ and $\Delta \mathrm{FEV}_{1}(\mathrm{r}=0.658, \mathrm{p}=0.015)$. There were differences in $\Delta \mathrm{IC}$ between patients whose $\mathrm{EMG}_{\text {paramax }}$ decreased during their admission and those whose $\mathrm{EMG}_{\text {para\%max }}$ increased (mean difference $0.50 \mathrm{l}$; $\mathrm{p}=0.003$ ) (Abstract S116 figure 1). There were no significant correlations between dyspnoea scores and $\Delta \mathrm{IC}$ or $\Delta \mathrm{EMG}_{\text {para\%max }}$.

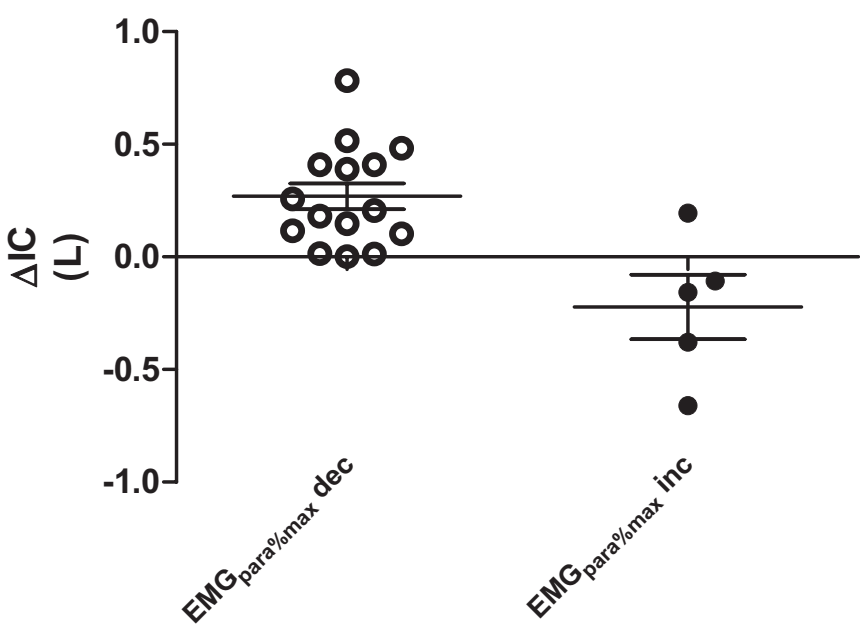

Abstract S116 Figure 1

Conclusion $\mathrm{DH}$ is a significant contributor to $\mathrm{NRD}$ in AECOPD. $\Delta \mathrm{EMG}_{\text {para\%max }}$ reflects changes in $\mathrm{DH}$ during hospital admission, but patient-reported dyspnoea does not indicate the degree of $\mathrm{DH}$, highlighting the limitations of dyspnoea scores. Changes in $\mathrm{DH}$ are correlated with changes in airflow obstruction. These data provide a physiological rationale for the utility of parasternal EMG as a noninvasive and non-volitional technique to track clinical change in AECOPD patients.

\section{S117 RESPIRATORY MUSCLE FATIGUE FOLLOWING EXERCISE IN PATIENTS WITH INTERSTITIAL LUNG DISEASE}

doi:10.1136/thoraxjnl-2011-201054b.117

D Elia, J L Kelly, D Martolini, E Renzoni, A K Boutou, A Chetta, M I Polkey, N S Hopkinson. NIHR Respiratory Biomedical Research Unit of Royal Brompton and Harefield NHS Trust and Imperial College, London, UK

Background Patients with interstitial lung disease (ILD) experience progressive breathlessness and exercise limitation. Although respiratory muscle fatigue has been investigated in healthy subjects and patients with COPD, it is unknown whether it occurs during exercise in ILD patients and, if so to what extent it is related to changes in dynamic lung volumes.

Methods Patients with ILD performed incremental, symptomlimited cycle ergometry with inspiratory capacity manoeuvres used to measure changes in end-expiratory lung volume (EELV). Twitch transdiaphragmatic pressure (TwPdi), in response to bilateral anterolateral magnetic phrenic nerve stimulation and twitch gastric pressure $\left(\mathrm{TwT}_{10} \mathrm{Pga}\right)$ in response to magnetic stimulation over the 10th thoracic vertebra were used to assess the development of fatigue.

Results Sixteen ILD patients (11 women) were studied. TwPdi did not differ significantly pre and post exercise (21.8 \pm 8 vs $20.2 \pm 8$ $\mathrm{cmH}_{2} \mathrm{O} ; \mathrm{p}=0.10$ ), while $\mathrm{TwT}_{10}$ Pga fell from $28.6 \pm 18$ to $25.2 \pm 14$ $\mathrm{cmH}_{2} \mathrm{O} \quad(\mathrm{p}=0.02) \quad$ (Abstract S117 figure 1). EELV fell from $2.18 \pm 0.651$ to $1.91 \pm 0.591$ following exercise $(p=0.04)$. The fall in $\mathrm{TwT10Pgas}$ correlated with peak $\mathrm{VO}_{2}(\mathrm{r}=-0.52, \mathrm{p}=0.041)$ increase in heart rate $(r=0.53 \mathrm{p}=0.032)$ and with the decrease of EELV during exercise $(r=0.57, p=0.021)$. Abdominal muscle fatiguers $(n=9,56 \%)$, defined as a $\geq 10 \%$ fall in TwT10Pga, had a fall in EELV of $22 \pm 22 \%$ compared to $0.7 \pm 8 \%$ in non-fatiguers $(p=0.016)$.
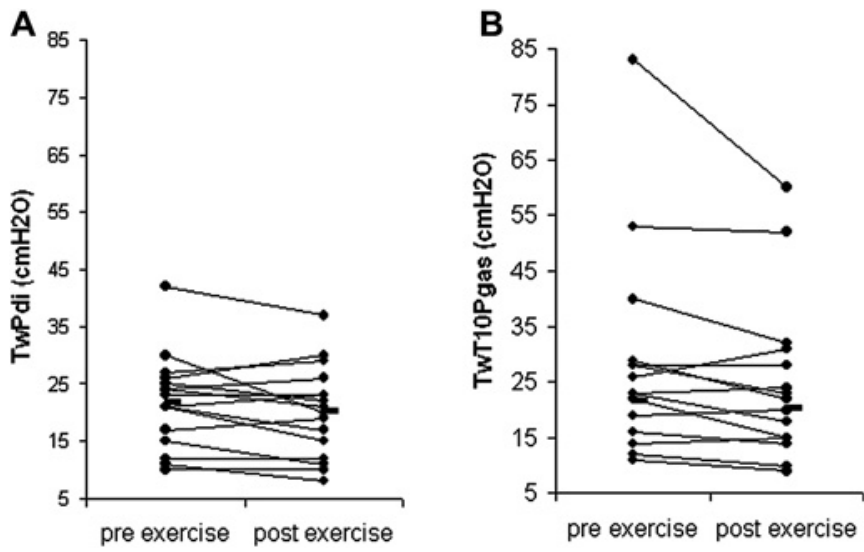

Abstract S117 Figure 1 Twitch transdiapragmatic $(A)$ and twitch gastric pressure (B) pressure before and after exercise.

Conclusion Abdominal muscle fatigue develops during exercise in ILD patients in association with increased expiratory muscle activity manifest by reduced EELV.

\section{S118 DIRECT VISUALISATION OF COLLATERAL VENTILATION IN COPD WITH HYPERPOLARISED GAS MRI}

doi:10.1136/thoraxjnl-2011-201054b.118

${ }^{1} \mathrm{H}$ Marshall, ${ }^{1} \mathrm{M} \mathrm{H}$ Deppe, ${ }^{1} \mathrm{~J}$ Parra-Robles, ${ }^{2} \mathrm{~S}$ Hillis, ${ }^{2} \mathrm{C}$ Billings, ${ }^{3} \mathrm{~S} \mathrm{R}$ Miller, ${ }^{3} \mathrm{~J} \mathrm{H}$ Watson, ${ }^{4} \mathrm{~J}$ Wolber, ${ }^{3} \mathrm{D}$ A Lipson, ${ }^{2} \mathrm{R}$ Lawson, ${ }^{1} \mathrm{~J} \mathrm{M}$ Wild. ${ }^{1}$ Academic Radiology, University of Sheffield, Sheffield, UK; ${ }^{2}$ Respiratory Medicine, Sheffield Teaching Hospitals NHS Trust, Sheffield, UK; ${ }^{3}$ GlaxoSmithKline, King of Prussia, Pennsylvania, USA; ${ }^{4}$ GE Healthcare, Amersham, UK

Introduction and Objectives Collateral ventilation is important in pathophysiology of Chronic Obstructive Pulmonary Disease (COPD), complicated pneumothorax, and bronchoscopic lung volume reduction surgery but limited observations of it in vivo have been attained. Current techniques capable of imaging collateral ventilation require monitoring over multiple breathing cycles and

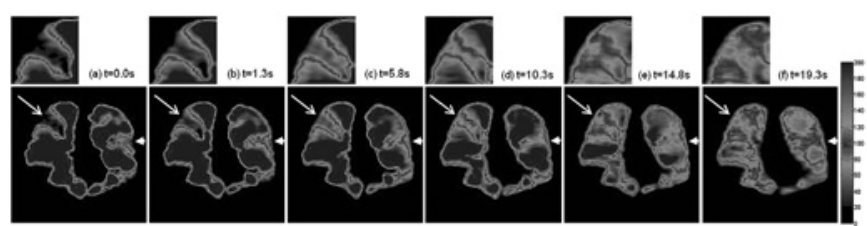

Abstract S118 Figure 1 Images tracking collateral ventilation in a COPD patient $(A-F)$, all displayed with the same colour-scale. 